\title{
Patient Alignment Estimation in Six Degrees of Freedom using a CT-scan and a Single X-ray Image
}

\author{
B. P. Selby ${ }^{1}$, G. Sakas ${ }^{1}$, S. Walter ${ }^{2}$, W.-D. Groch ${ }^{3}$, U. Stilla ${ }^{4}$ \\ ${ }^{1}$ Cognitive Computing and Medical Imaging, Fraunhofer IGD Darmstadt, Germany \\ ${ }^{2}$ Medcom GmbH, Darmstadt, Germany \\ ${ }^{3}$ Fachbereich Informatik, University of Applied Sciences Darmstadt, Germany \\ ${ }^{4}$ Photogrammetry and Remote Sensing, Technische Universitaet Muenchen, Germany \\ pselby@medcom-online.de
}

\begin{abstract}
Particle beam treatment allows accurate dose delivery onto carcinogen tissue. The reachable accuracy is limited by patient alignment errors relative to the beam source. Errors can be corrected manually or by automatic comparison of two X-ray images to a CT-scan but correction mostly does not cover all degrees of freedom (DoF). In this contribution we present a solution that makes use of one X-ray image and computes full 6 DoF alignment correction by gray value based comparison to a $\mathrm{CT}$. By using regions of interest, we are able to increase performance and reliability.
\end{abstract}

\section{Introduction}

A rapid growing number of commercial health centers exploit the advances of particle beams. Particle beam based radiotherapy allows accurate application of the treatment dose onto carcinogen tissue with accuracy less than $1.0 \mathrm{~mm}$ and therefore efforts accurate alignment of the patient in the facility [1]. Tracking external markers or patient fixation does not suffice the setup precision requirements. It is common practice in image guided radiotherapy to align patients manually in the device by visual evaluation of X-ray images and digital reconstructed radiographs (DRRs) to estimate misalignments [2]. The CT data recorded during the treatment planning serves as reference position of the relevant body part. $\mathrm{X}$-ray images, acquired before treatment from within the radiation device, reflect the real patient alignment.

During the time consuming and error prone manual procedure, the alignment of the respective body region may change, leading to degradation of the treatment results. Additionally, manual alignment cannot be done for six degrees of freedom (DoF), because rotational misalignments can hardly be detected visually in the $2 \mathrm{D}$ images.

To overcome these problems automatic procedures can be used for determination and correction of patient alignment errors. As described in $[3,4]$, this can be done by registration of two $2 \mathrm{D}$ images to a reference CT series. For example 
$2 \mathrm{kV}$ X-ray images can be registered with the projections of a CT projected to 2D space. However, using two X-ray images implies twice the exposure applied to the patient and is coupled with much higher expenses, than if just a single $\mathrm{X}$-ray image would be used.

To overcome these drawbacks, our approach implements a gray-value distribution based image comparison between a single X-ray image acquired from within the treatment device and digital reconstructed radiographs that we generate on demand from a CT volume for hypothetical patient positions.

\section{Methods}

The automatic procedure consists of the following steps (Fig. 1 left): Generation of a suitable DRR for an estimated patient position, gray-value based comparison of the DRR to the region of interest (ROI) in the X-ray image, maximization of the similarity measure through repeated DRR generation with 6 free parameters (rigid transformation: 3 shifts, 3 rotations) and finally calculation of correctional values for the patient alignment (Fig. 2 right).

\subsection{Projection of the CT}

As we have to perform several iterations of DRR renderings, we use a raytracing algorithm optimized for our purpose. We do not intend to generate high quality DRRs, but DRRs that are adapted for fast rendering and comparison with an $\mathrm{X}$-ray image.

When scattering is neglected, the intensity of an X-ray passing through the respective object is given by the Radon transform

$$
I=I_{0} \cdot \exp \left(-\int_{-\infty}^{\infty} f(x, m x+b)\right) d x
$$

where $I_{0}$ is the intensity of the X-ray at the source and the integral is the line integral along the ray. As we do not know the source energy, we just choose $I_{0}$ to normalize the expression in (1) to a resulting intensity range of $I \in[0.0,1.0]$,

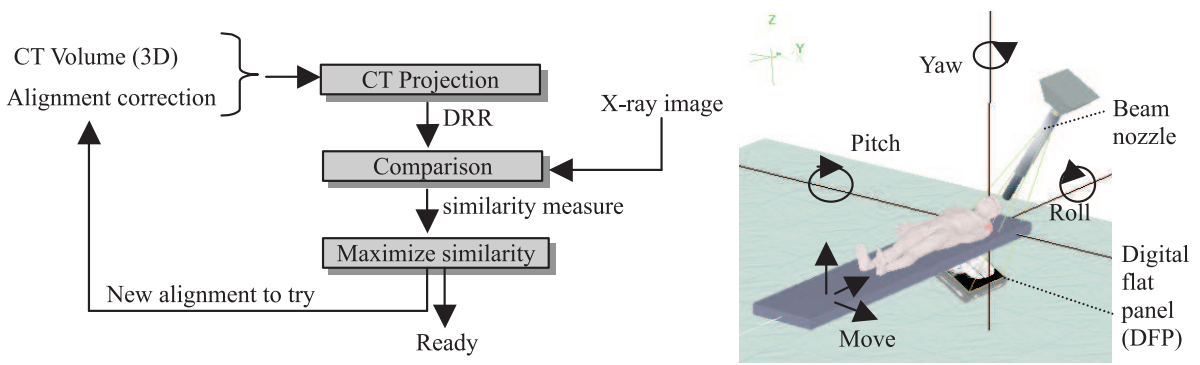

Fig. 1. Correction procedure (left) and treatment geometry (right) 
which can be scaled to discrete 12 bit pixel values. In our case, the object the ray runs through is the $\mathrm{CT}$ volume. We can increase performance by precalculating and storing $f$ for each voxel. $f$ can be obtained by transformation of the CT's Hounsfield values to their attenuation factors. Computation of I for each pixel resided in a plane corresponding to the geometric setup of the digital flat panel (DFP) for the X-ray image gives the DRR image. For further performance enhancement, we transfer the area of a ROI, if defined in the X-ray image by the radiotherapist, to it's assumed position in the DRR, using the preliminary alignment of the patient. We build-up a bit-mask in the DRR image domain defining the pixels contained in the ROI and perform the rendering only for the respective pixels.

\subsection{Comparison of the DRR to the X-ray Image}

There exists a wide range of gray value based image comparators. As methods like crosscorrelation or usage of difference images are not suitable for images that differ in much more aspects than contrast and intensity, we decided to use mutual information as similarity measure [4]. The Mutual Information is given by

$$
\mathrm{MI}(A, B)=\iint P(a \mid b) \ln (P(a \mid b))-\int P(a) \ln P(a)-\int P(b) \ln (b)
$$

where $P$ is the probability of gray value $a$ from image $A$ (X-ray image) or of gray value $b$ from image $B$ (DRR). The integrals mean integration over all existing gray values. However, we suppress high and low gray values to improve performance and to reduce the influence of features coming from corrupt pixel values or metallic objects, as fixation equipment. Therefore we only integrate over the center of the joint histogram given by $P(a \mid b)$ that consists of $80 \%$ of the image pixels. This also assures, that extreme pixels that do rarely match, are not taken into account. If the radiotherapist has defined a ROI, we only use the gray values residing this area to compute the similarity, so only the relevant image parts are used (Fig. 2).

\subsection{Maximization of the Similarity Measure and Alignment Correction}

To find the rigid transformation $T$ for the current patient pose we minimize the negative similarity value given in $(3)$ :

$$
\mathrm{OPT}=\min (-M I(A, B(T)))
$$

where $B$ is the projected CT. The elements of $T$ serve as free parameters in the optimization and for each change of $T$ we need to reproject the CT-scan.

We minimize by the downhill simplex approach [5] for 6 parameters $(3$ shifts and 3 rotations). The minimization procedure is ready, as soon as the $6 \mathrm{D}$ simplex of 7 sextupels becomes smaller than $\sigma_{\text {dist }}=0.25 \mathrm{~mm}$ or $\sigma_{\text {angle }}=0.25^{\circ}$. 
Table 1. Correction results for different alignment errors

\begin{tabular}{llll}
\hline Dataset & $\begin{array}{l}\text { Intended alignment error } \\
\text { (Rotation/Shift) }\end{array}$ & $\begin{array}{l}\text { Error after correction } \\
\text { (Rotation/Shift) }\end{array}$ & $\begin{array}{l}\text { Calculation time } \\
\text { (in seconds) }\end{array}$ \\
\hline Skull & $1^{\circ} / 1 \mathrm{~mm}$ & $0.4^{\circ} / 0.7 \mathrm{~mm}$ & 53 \\
Skull & $2^{\circ} / 5 \mathrm{~mm}$ & $0.4^{\circ} / 0.6 \mathrm{~mm}$ & 42 \\
Skull & $3^{\circ} / 10 \mathrm{~mm}$ & $0.4^{\circ} / 0.9 \mathrm{~mm}$ & 61 \\
Pelvis & $1^{\circ} / 1 \mathrm{~mm}$ & $0.3^{\circ} / 1.1 \mathrm{~mm}$ & 82 \\
Pelvis & $2^{\circ} / 5 \mathrm{~mm}$ & $0.4^{\circ} / 1.0 \mathrm{~mm}$ & 85 \\
Pelvis & $3^{\circ} / 10 \mathrm{~mm}$ & $0.6^{\circ} / 1.8 \mathrm{~mm}$ & 97 \\
Skull & $2^{\circ} / 5 \mathrm{~mm}(4 \mathrm{~mm}$ along X-ray) & $0.5^{\circ} / 2.9 \mathrm{~mm}$ & 55 \\
Pelvis & $2^{\circ} / 5 \mathrm{~mm}(4 \mathrm{~mm}$ along X-ray) & $0.7^{\circ} / 4.3 \mathrm{~mm}$ & 121 \\
\hline
\end{tabular}

The resulting transformation $\mathrm{T}$ corresponds to the difference between the current patient alignment as seen by the DFP and the reference alignment given by the simulated spacial position of the $\mathrm{CT}$ volume and the Isocenter in $\mathrm{CT}$ coordinates in the treatment device. To receive the correction for the alignment parameters of the patient table we finally transfer the inverse transformation inv $(T)$ to coordinates for the table that realign the patient.

\section{Results}

Tests have been performed using a CT dataset (295 slices, $0.8 \mathrm{~mm}$ slice distance) of a human scull phantom (Fig. 3) and a CT dataset (300 slices, $1.0 \mathrm{~mm}$ slice distance) of an anathomic pelvis phantom. We produced intended alignment errors as can be expected in real treatment situations and used respective X-ray images taken from within a tratment device to computed the correction (Tab. $3)$.

Using a ROI, the results are slightly more inaccurate in general, but the algorithm is able to find a correction in much shorter time (inverse proportional

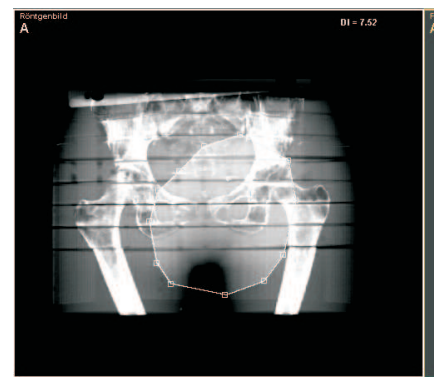

a

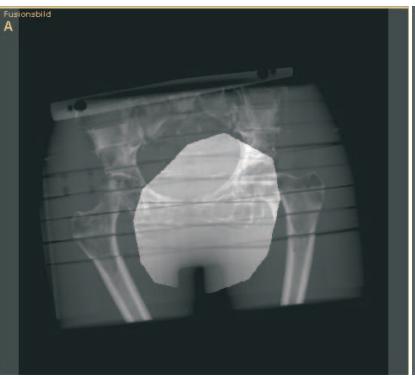

b

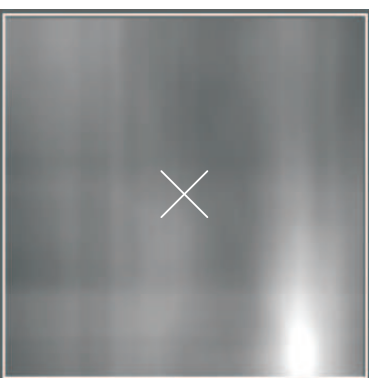

Fig. 2. X-ray image of pelvis phantom with ROI (a); overlaid with respective DRR (b); Mutual Information for $20 \mathrm{~cm}$ x $20 \mathrm{~cm}$ transformations (current MI value at center) 
to the ROI area) and it shows to be more stable if disturbing features can be excluded.

\section{Discussion}

Automatic image based position correction is mandatory for efficient operation of a commercial particle beam treatment facility. The results show that our automatic position correction procedure is able to improve or verify the initial patient alignment. The number of X-ray images can be reduced to one X-ray image, whereas manual setup would need several iterations and pairs of X-ray images. In this way the X-ray dose applied onto the patient can be reduced. The acceleration of the setup procedure assures a contemporary radiation treatment with fewer changes of the patient alignment before treatment and additionally allows a higher patient throughput. However, compared to the automatic method proposed in [3], which makes use of 2 images, the single X-ray image approach suffers from lower accuracy if large translations along the X-ray beam axis occur (Tab. 1: $4 \mathrm{~mm}$ along X-ray). That is because using a single X-ray image this translation value highly depends on correct determination of the image scale,

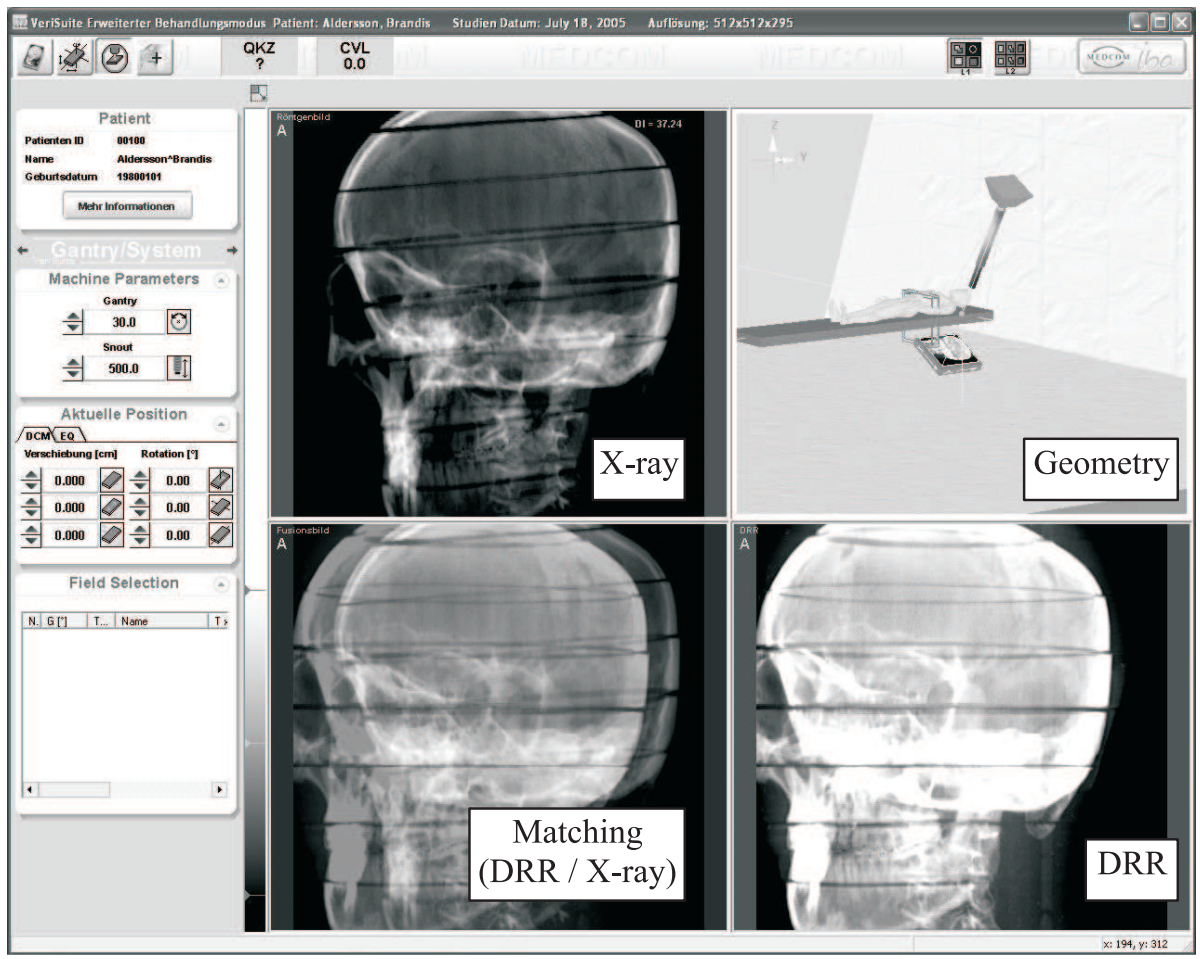

Fig. 3. Alignment correction application with images of a human scull phantom 
which is, depending on the geometric setup of the X-ray equipment, not very sensitive to changes in translations of the patient. But, as higher accuracy could be achieved using higher resolution CT-scans and a modified alignment of the $\mathrm{X}$-ray equipment, it should be possible to overcome this problem and to benefit from the advantages using only a single DFP.

\section{References}

1. Verhey LJ, Goitein M, McNulty P, et al. Precise positioning of patients for radiation therapy. Int J Radiat Oncol Biol Phys. 1982;8(2):289-94.

2. Thilmann C, Nill S, Tücking T, et al. Correction of patient positioning errors based on in-line cone beam CTs: Clinical implementation and first experiences. Int J Radiat Oncol Biol Phys. 2005;63(1):550-1.

3. Selby B, Sakas G, Walter S, et al. Detection of pose changes for spatial objects from projective images. In: Photogrammetric Image Analysis, The International Archives of the Photogrammetry, Remote Sensing and Spatial Information Sciences; 2007. p. 105-10.

4. Clippe S, Sarrut D, Malet C, et al. Patient setup error measurement using 3D intensity-based image registration techniques. Int $\mathrm{J}$ Radiat Oncol Biol Phys. 2003;56(1):259-65.

5. Press WH, Teukolsky SA, Vetterling WT, et al. Numerical Recipes in C. vol. 2. Cambridge: Cambridge University Press; 1992. 